\section{Impurity in ${ }^{68}$ Ga-Peptide Preparation Using Processed Generator Eluate}

TO THE EDITOR: Zhernosekov et al. (1) have published a method for processing generator eluate from ${ }^{68} \mathrm{Ge} /{ }^{68} \mathrm{Ga}$ generators for medical applications, and currently this method is widely used for clinical preparations, especially of ${ }^{68} \mathrm{Ga}$-labeled $1,4,7,10$-tetraazacyclododecane- $N, N^{\prime}, N^{\prime \prime}, N^{\prime \prime \prime}$-tetraacetic acid (DOTA)D-Phe ${ }^{1}, \mathrm{Tyr}^{3}$-octreotide (DOTATOC) and other somatostatin analogs. The method is based on preconcentration of ${ }^{68} \mathrm{Ga}$ ions in the eluate on a cation exchanger followed by selective washing and subsequent elution using hydrochloric acid/acetone solutions. This method removes unwanted metallic impurities and reduces the volume, as is especially suitable for radiolabeling of small peptides such as DOTATOC. We investigated this method in an attempt towards developing a fully automated system. During our validation steps of this method, we observed an unknown peak in the ultraviolet trace $(220 \mathrm{~nm})$ of the high-performance liquid chromatograms of ${ }^{68} \mathrm{Ga}$-labeled peptides increasing over time with prolonged storage of the hydrochloric acid/acetone solutions. The peak eluted closely to DOTATOC or DOTA-D-Phe ${ }^{1}, \mathrm{Tyr}^{3}$ octreotate used for radiolabeling when analyzed under standard acetonitrile/0.1\% trifluoroacetic acid/water gradient high-performance liquid chromatography (HPLC) conditions. By means of gas chromatography mass spectrometry, we finally identified the peak to be 4-methyl-3-penten-2-on (mesityloxide), a condensation product of acetone. Apparently, this impurity and the ${ }^{68} \mathrm{Ga}$-labeled peptide have comparable properties on the reversed-phase (C-18) material used both for purification after synthesis and for quality control by HPLC. Because of the high boiling point $\left(129^{\circ} \mathrm{C}\right)$ of this compound, the peak was detected even when prolonged heating was applied, and column purification failed to remove it. We could show that the amount of mesityloxide formed is dependent on storage time and storage conditions of the hydrochloric acid/acetone stock solutions. Storage at room temperature and exposure to light resulted in formation of milligram amounts, and up to $0.7 \mathrm{mg}$ was detected in a final volume corresponding to a patient preparation. If the mixtures were prepared freshly or stored with protection from light at $-20^{\circ} \mathrm{C}$, this amount was below $10 \mu \mathrm{g}$. Mesityloxide is not a highly toxic compound, and median lethal dose in rats $(1.12 \mathrm{mg} / \mathrm{kg}(2))$ is comparable to that of acetone. However, as no limits for parenteral preparations are available in relevant documents such as the U.S. Pharmacopeia, the amount should be limited; a reasonably achievable limit seems to be $50 \mu \mathrm{g}$ per maximum volume injected. We strongly recommend all users of this method to include into their quality control protocols a quantification of this impurity by means of HPLC-ultraviolet detection or gas chromatography. Additionally, careful validation of preparation and storage of hydrochloric acid/ acetone solutions has to be performed. Alternative preparation methods avoiding the use of hydrochloric acid/acetone mixtures have been described $(3,4)$ and may also be envisaged.

COPYRIGHT @ 2010 by the Society of Nuclear Medicine, Inc.

\section{REFERENCES}

1. Zhernosekov KP, Filosofov DV, Baum RP, et al. Processing of generator-produced ${ }^{68} \mathrm{Ga}$ for medical application. $J$ Nucl Med. 2007;48:1741-1748.

2. Sigma-Aldrich safety data sheet: mesityloxide. Version 3.0; revision date, August 22, 2009. Available at: http://www.arkema-inc.com/msds/1032.pdf. Accessed December 16, 2009.

3. Velikyan I, Beyer GJ, Långström B. Microwave-supported preparation of ${ }^{68} \mathrm{Ga}$ bioconjugates with high specific radioactivity. Bioconjug Chem. 2004;15:554560 .

4. Decristoforo C, Knopp R, von Guggenberg E, et al. A fully automated synthesis for the preparation of ${ }^{68} \mathrm{Ga}$-labelled peptides. Nucl Med Commun. 2007;28: $870-875$.

Milos Petrik

Meltem Ocak

Marco Rupprich

Clemens Decristoforo*

*Clinical Department of Nuclear Medicine

University of Innsbruck

Anichstrasse 35

A-6020 Innsbruck, Austria

E-mail: clemens.decristoforo@uki.at

DOI: $10.2967 /$ jnumed.109.070953

REPLY: I thank Dr. Petrik and colleagues for their interest in our work $(1,2)$. Our method of processing ${ }^{68} \mathrm{Ga}$ eluates indeed has several advantages allowing instant kit-type synthesis procedures. First, it purifies this generator-derived positron emitter of metals such as zinc, iron, and titanium, significantly improving the yield of subsequent labeling reactions. Second, it lowers the initial breakthrough of ${ }^{68} \mathrm{Ge}$ by more than 4 orders of magnitude down to negligible levels, thus minimizing concerns about radiopharmaceutical contamination by this long-lived radionuclide. Third, it reduces the total volume of the purified ${ }^{68} \mathrm{Ga}$ fraction to $0.4 \mathrm{~mL}$, and finally, it removes excess hydrochloric acid originating from the generator eluate. Recent improvements also demonstrate that the intermediate adsorption of purified ${ }^{68} \mathrm{Ga}$ on the small cation exchange cartridge opens the door toward synthesis of lipophilic ${ }^{68} \mathrm{Ga}$-compounds in nonaqueous solvents (3).

Moreover, the removal of ${ }^{68} \mathrm{Ge}$ represents an inherent safety procedure, as it automatically eliminates any unexpected leakage from the generator. Such a sudden dramatic problem, eventually induced by defects and incorrect handling of the generator, is neither directly seen nor avoided by using fractionation as an approach toward eluate postprocessing $(4,5)$.

Consequently, postprocessing ${ }^{68} \mathrm{Ga}$ eluates $(1,2)$ has become a well-adopted procedure, applicable both to $0.1 \mathrm{~N}$ and to $0.6 \mathrm{~N}$ $\mathrm{HCl}$ eluate concentrations (for $\mathrm{TiO}_{2}$ - and $\mathrm{SnO}_{2}$-based generators, respectively).

In the cation exchange-based processing chemistry and subsequent (for example) ${ }^{68} \mathrm{Ga}$-DOTA-octreotide synthesis and product purification steps, nontoxic ingredients such as Millipore water, saline, acetone, and ethanol are involved exclusively. Preparation and storage of these solvents and acetone $/ \mathrm{HCl}$ mixtures in the context of application of ${ }^{68} \mathrm{Ga}$-labeled radiopharmaceuticals for patient diagnoses is well defined as the responsibility of the 
qualified persons in installation qualification, operational qualification, and standard operating procedure protocols.

Consequently, the solutions have to be prepared from highpurity batches, have to be kept in closed vials, and have to be refrigerated or stored at $-20^{\circ} \mathrm{C}$ for a limited time only. If not, the sterility of the Millipore water and the saline solutions is not guaranteed, the composition of the acetone/ $\mathrm{HCl}$ mixtures will change because of the low boiling points of the compounds, and the acetone in acidic media will undergo an aldol addition reaction forming 4-methyl-3-penten-2-on. We appreciate the effort of Petrik et al. to "finally" identify this well-known product, confirming the standard education of chemistry students (6).

If it was the intention of the present letter to the editor to reflect the relevancy of creating and following standard operating procedures for the synthesis of radiopharmaceuticals, we completely agree. Regarding the nontoxic compound 4-methyl-3penten-2-on, Petrik et al. correctly state that its formation is negligible if acetone/ $\mathrm{HCl}$ mixtures are stored with protection from light at $-20^{\circ} \mathrm{C}$ or if freshly prepared mixtures are used.

\section{REFERENCES}

1. Zhernosekov KP, Filosofov DV, Baum RP, et al. Processing of generator-produced ${ }^{68} \mathrm{Ga}$ for medical application. J Nucl Med. 2007;48:1741-1748.

2. Asti M, De Pietria G, Fraternalia A, et al. Validation of ${ }^{68} \mathrm{Ge} /{ }^{68} \mathrm{Ga}$ generator processing by chemical purification for routine clinical application of ${ }^{68} \mathrm{Ga}$ DOTATOC. Nucl Med Biol. 2008;35:721-724.

3. Zoller F, Riss PJ, Montforts F-P, Rösch F. Efficient post-processing of aqueous generator eluates facilitates ${ }^{68} \mathrm{Ga}$-labelling under anhydrous conditions. Radiochim Acta. 2010. In press.

4. Velikyan I, Beyer GJ, Långström B. Microwave-supported preparation of ${ }^{68} \mathrm{Ga}$ bioconjugates with high specific radioactivity. Bioconjug Chem. 2004;15:554-560.

5. Decristoforo C, Knopp R, von Guggenberg E, et al. A fully automated synthesis for the preparation of ${ }^{68} \mathrm{Ga}-$ labelled peptides. Nucl Med Commun. 2007;28:870875 .

6. Schwetlick K. Organikum. 22nd ed. Weinheim, Germany: Wiley-VCH; 2004.

\author{
Frank Rösch \\ University of Mainz \\ F.-Strassmann-Weg 2 \\ D-55128 Mainz, Germany \\ E-mail:frank.roesch@uni.mainz.de
}

DOI: 10.2967/jnumed.109.071084

Side Effects Profile in Humans of ${ }^{11} \mathrm{C}-(+)$-PHNO, a Dopamine $\mathrm{D}_{2 / 3}$ Agonist Ligand for PET

TO THE EDITOR: ${ }^{11} \mathrm{C}-(+)-4$-propyl-9-hydroxynaphthoxazine $((+)$-PHNO) is a new PET ligand developed by our group. Binding assays show that (+)-PHNO displays high affinity and selectivity for the $\mathrm{D}_{2}$ receptor (1). Recently, it has been noted that ${ }^{11} \mathrm{C}-(+)-\mathrm{PHNO}$ has a preferential affinity and selectivity in vivo for the $\mathrm{D}_{3}$ receptors $(2)$. Because ${ }^{11} \mathrm{C}-(+)$-PHNO is an agonist radiotracer for $D_{2}$ and $D_{3}$, it is likely to produce pharmacologic effects, in contrast to antagonist radiotracers. We reviewed all ${ }^{11} \mathrm{C}-(+)$-PHNO consecutive scans obtained in our PET center for side effects. Mass injected $(\mu \mathrm{g})$, subjects' weight $(\mathrm{kg})$, and dose $(\mu \mathrm{g} / \mathrm{kg})$ were included in the analysis. Side effects were recorded on the basis of the subjects' self-report either during or right after finalization of the scan. A physician was available at all times to confirm and treat any possible side effects. Side effects were coded as 0 (no effect), 1 (nausea), or 2 (vomiting), based on our early experience with ${ }^{11} \mathrm{C}-(+)-\mathrm{PHNO}(3)$. Odds ratios (ORs) were calculated using logistic regression analyses to investigate the relationship between dose, mass, and effects.

The number of reviewed ${ }^{11} \mathrm{C}-(+)$-PHNO scans totalled 486. Injected mass ranged from 0.85 to $5.56 \mu \mathrm{g}$, with a mean of $2.30 \mu \mathrm{g}$ $(\mathrm{SE}, 0.024 \mu \mathrm{g}$ ). Injected doses ranged from 0.01 to $0.08 \mu \mathrm{g} / \mathrm{kg}$, with a mean of $0.03 \mu \mathrm{g} / \mathrm{kg}$ (SE, $0.0004 \mu \mathrm{g} / \mathrm{kg}$ ). No effect was present in $84.6 \%$ of the scans reviewed; nausea was present in $14.3 \%$, and vomiting in $1.1 \%$. Symptoms arose $3-5$ min after the injection and subsided within 7-12 min in all cases. In none of the cases was any medical action required.

In a logistic regression model including all subjects, nausea was significantly predicted by dose (Wald $=21.70, P<0.001$, OR 1.99 ) and mass (Wald $=16.319, P<0.001$, OR $=2.826$ ), and vomiting was significantly predicted by dose (Wald $=7.31$, $P<0.007, \mathrm{OR}=2.66$ ) but not by mass injected (Wald $=0.694$, $P=0.405$, OR $=1.810)$. When only drug-free volunteers were analyzed $(n=209)$, no effect was present in $79.8 \%$ of the cases, nausea was present in $18.7 \%$, and vomiting in $1.5 \%$. In a logistic regression model including only drug-free volunteers, nausea was significantly predicted by dose (Wald $=6.98, P<0.008$, OR 1.54) and mass (Wald $=11.981, P=0.001$, OR $=2.843$ ). Vomiting was predicted at a trend level by dose (Wald $=3.33$, $P<0.06$, OR 2) but not by mass (Wald $=0.105, P=0.746$, $\mathrm{OR}=1.303)$. When only antipsychotic-treated participants were analyzed $(n=66)$, no effect was present in $97 \%$ of the cases, nausea was present in $3 \%$, and no vomiting was present in any. In a logistic regression model including only these subjects, nausea was significantly predicted neither by dose (Wald $=2.25, P<$ 0.13 ) nor by mass (Wald $=0.000, P=0.99$ ). In all cases, when an injected dose of $0.029 \mu \mathrm{g} / \mathrm{kg}$ or less was selected, there was no relationship between dose and nausea.

The side effects reported in this study are consistent with the expected agonism at the $\mathrm{D}_{2}$ - and $\mathrm{D}_{3}$-receptor (4-7).

We conclude that doses of ${ }^{11} \mathrm{C}-(+)-\mathrm{PHNO}$ of $0.029 \mu \mathrm{g} / \mathrm{kg}$ or less are highly unlikely to produce any side effects in humans and that ${ }^{11} \mathrm{C}-(+)-\mathrm{PHNO}$ is a safe agonist radiotracer for PET in human studies of health and disease.

\section{ACKNOWLEDGMENTS}

We acknowledge the scientists and technicians from the PET center who carried out the ${ }^{11} \mathrm{C}-(+)-\mathrm{PHNO}$ scans. This work is supported in part by the CIHR and the OMHF New Investigator Awards.

\section{REFERENCES}

1. Wilson AA, McCormick P, Kapur S, et al. Radiosynthesis and evaluation of $\left[{ }^{11} \mathrm{C}\right]-$ (+)-4-propyl-3,4,4a,5,6,10b-hexahydro-2H-naphtho[1,2-b][1,4]oxazin-9-ol as a potential radiotracer for in vivo imaging of the dopamine $\mathrm{D}_{2}$ high-affinity state with positron emission tomography. J Med Chem. 2005;48:4153-4160.

2. Narendran R, Slifstein M, Guillin $O$, et al. Dopamine $\left(D_{2 / 3}\right)$ receptor agonist positron emission tomography radiotracer $\left[{ }^{11} \mathrm{C}\right]-(+)-\mathrm{PHNO}$ is a $\mathrm{D}_{3}$ receptor preferring agonist in vivo. Synapse. 2006;60:485-495.

3. Willeit M, Ginovart N, Kapur S, et al. High-affinity states of human brain dopamine $\mathrm{D}_{2 / 3}$ receptors imaged by the agonist $\left[{ }^{11} \mathrm{C}\right]-(+)$-PHNO. Biol Psychiatry. 2006;59:389-394.

4. Yoshida N, Yoshikawa T, Hosoki K. A dopamine $\mathrm{D}_{3}$ receptor agonist, 7-OHDPAT, causes vomiting in the dog. Life Sci. 1995;57:PL347-PL350. 
5. Grandas F, Quinn N, Critchley P, Rohan A, Marsden CD, Stahl SM. Antiparkinsonian activity of a single oral dose of PHNO. Mov Disord. 1987;2: 47-51.

6. Coleman RJ, Quinn NP, Traub M, Marsden CD. Nasogastric and intravenous infusions of (+)-4-propyl-9-hydroxynaphthoxazine (PHNO) in Parkinson's disease. J Neurol Neurosurg Psychiatry. 1990;53:102-105.

7. Cedarbaum JM, Clark M, Toy LH, Green-Parsons A. Sustained-release (+)-PHNO [MK-458 (HPMC)] in the treatment of Parkinson's disease: evidence for tolerance to a selective $\mathrm{D}_{2}$-receptor agonist administered as a long-acting formulation. Mov Disord. 1990;5:298-303.

\author{
Romina Mizrahi* \\ Sylvain Houle \\ Irina Vitcu \\ Alvina Ng \\ Alan A Wilson \\ *PET Centre, CAMH \\ University of Toronto \\ 250 College St. \\ Toronto, ON, M5T 1R8, Canada \\ E-mail: romina.mizrahi@camhpet.ca
}

DOI: $10.2967 /$ jnumed.109.072314

\section{The Twilight Saga of Insulin Administration in Hyperglycemic Patients Undergoing ${ }^{18}$ F-FDG PET}

TO THE EDITOR: Roy et al. recently published a study adopting a standardized protocol of intravenous insulin administration to reduce glycemia in diabetic cancer patients undergoing ${ }^{18} \mathrm{~F}$-FDG PET (1). The authors claimed that the pretest intravenous insulin injection in diabetic patients is a realistic approach. However, several issues deserve further exploration before this standardized insulin protocol can be incorporated into daily PET practice.

In clinical tumor imaging, hyperglycemia has a recognized adverse effect on the quality of ${ }^{18}$ F-FDG PET images because of competitive inhibition of ${ }^{18} \mathrm{~F}-\mathrm{FDG}$ uptake by glucose. Although insulin can be used as a glycemia-reducing agent, arbitrary prescription of insulin before ${ }^{18} \mathrm{~F}$-FDG injection may exacerbate muscular ${ }^{18} \mathrm{~F}$-FDG uptake and compromise tumor uptake, thus curtailing image interpretability (2). According to the study results of Roy et al., ${ }^{18}$ F-FDG PET image quality was barely adequate in $75 \%$ of patients receiving insulin. This means every 1 of 4 scans must be repeated. Repeating a study is not a cost-benefit if the PET center does not have its own on-site cyclotron. Rescheduling is inconvenient to the patients and bothersome to the center staff. The set point to prescribe insulin in the study protocol of Roy et al. might account for their poor image quality. The Society of Nuclear Medicine recommends rescheduling the examination if the patient's blood glucose level is greater than $8.3-11.1 \mathrm{mmol} / \mathrm{L}$ (150-200 mg/dL) (3). The European Association of Nuclear Medicine also advises that an ${ }^{18}$ F-FDG PET study should not be performed when the blood glucose level exceeds $11.1 \mathrm{mmol} / \mathrm{L}$ (4). If cancelling an examination or rescheduling an appointment is not feasible, we suggest the use of intravenous insulin at a blood glucose level of more than $11.1 \mathrm{mmol} / \mathrm{L}$, instead of the $10.0 \mathrm{mmol} / \mathrm{L}$ stated by Roy et al. Additionally, we encourage hyperglycemic patients to have a temperate walk after insulin injection to reduce muscular uptake. In this way, the proportion of images of adequate quality would improve.
In their study, less favorable image quality was found with more glycemic reduction after insulin administration, and no significant correlation was observed between muscular uptake and parameters such as initial glycemia, total insulin dose, and number of insulin doses. Hence, the extent of glycemic reduction is not predictable and the chance of study failure is unavoidable. The implication is that we cannot select which hyperglycemic patient is suitable for insulin use. We also cannot apply the correct insulin dose to manage glycemic reduction before imaging. These phenomena can be explained by nonuniform insulin sensitivity among hyperglycemic patients. Therefore, Roy's standardized insulin protocol, an illogical practice such as sliding-scale insulin (5), is a problematic recipe for glycemic control in hyperglycemic patients undergoing ${ }^{18} \mathrm{~F}$-FDG PET studies. Sliding-scale insulin is also associated with poorer glycemic control, a harmful rollercoaster effect between hyperglycemic and hypoglycemic episodes, and increased risks of hypoglycemia, as occurred in 6 patients $(9.5 \%)$ in the study of Roy et al. Thus, their standardized insulin protocol might be a risky strategy.

To obtain a useful diagnostic image of ${ }^{18} \mathrm{~F}$-FDG PET, one should ensure that the patient has fasted overnight and has a blood glucose level of less than $8.3 \mathrm{mmol} / \mathrm{L}$ in the early morning on the day of the PET scan. A good method is to do a "practice run" by checking the patient's blood glucose levels for at least $3 \mathrm{~d}$ before the ${ }^{18} \mathrm{~F}-\mathrm{FDG}$ PET appointment (6). If the morning blood glucose level is persistently higher than $8.3 \mathrm{mmol} / \mathrm{L}$, the scheduler needs to recognize this problem well before the scan appointment and request that the diabetologist manage the glycemic status by basal and nutritional insulin therapy with a supplemental insulin regimen (7). To avert the possibility of irreversibly unreadable images, hypoglycemia, and transcellularshift hypokalemia, before establishing specific guidelines for using insulin in hyperglycemic patients undergoing ${ }^{18} \mathrm{~F}-\mathrm{FDG}$ PET we should have the patients fast and we should not administer additional insulin.

\section{REFERENCES}

1. Roy F-N, Beaulieu S, Boucher L, Bourdeau I, Cohade C. Impact of intravenous insulin on ${ }^{18}$ F-FDG PET in diabetic cancer patients. J Nucl Med. 2009;50:178183

2. Coleman RE. Clinical PET in oncology. Clin Positron Imaging. 1998;1:15-30.

3. Delbeke D, Coleman RE, Guiberteau MJ, et al. Procedure guideline for tumor imaging with ${ }^{18} \mathrm{~F}$-FDG PET/CT 1.0. J Nucl Med. 2006;47:885-895.

4. Bombardieri E, Aktolun C, Baum RP, et al. FDG-PET: procedure guidelines for tumour imaging. Eur J Nucl Med Mol Imaging. 2003;30:BP115-BP124.

5. Umpierrez GE, Palacio A, Smiley D. Sliding scale insulin use: myth or insanity? Am J Med. 2007;120:563-567.

6. Hamblen SM, Lowe VJ. Clinical ${ }^{18}$ F-FDG oncology patient preparation techniques. J Nucl Med Technol. 2003;31:3-7.

7. Clement S. Better glycemic control in the hospital: beneficial and feasible. Cleve Clin J Med. 2007;74:111-112, 114-120.

\author{
Yuh-Feng Lin \\ Fu-Chiu Yu \\ Jainn-Shiun Chiu* \\ *Taipei Medical University-Shuang Ho Hospital \\ No. 291, Zhongzheng Rd. \\ Zhonghe City, Taipei County 235, Taiwan \\ E-mail:shiunkle@mail2000.com.tw
}

DOI: 10.2967/jnumed.109.070185 
REPLY: We thank Dr. Chiu et al. for their interest in our recent investigation on the impact of intravenous insulin on ${ }^{18} \mathrm{~F}-\mathrm{FDG}$ PET in diabetic cancer patients (1), and we would like to address their concerns.

The decision to use a $10.0 \mathrm{mmol} / \mathrm{L}$ threshold for insulin administration was based on the expected limited influence of this glycemia level on ${ }^{18} \mathrm{~F}$-FDG uptake and on the fact that it approaches the $\mathrm{Km}$ value of glucose transporter 3 (2). The Society of Nuclear Medicine guidelines state that most institutions reschedule the patient if the blood glucose level is greater than $8.3-11.1 \mathrm{mmol} / \mathrm{L}$ (3). The European Association of Nuclear Medicine does not recommend proceeding with an ${ }^{18} \mathrm{~F}-\mathrm{FDG}$ PET study when the glucose level in the blood exceeds $11.1 \mathrm{mmol} / \mathrm{L}$ (4). Choosing a higher threshold could reduce PET sensitivity. Choosing a lower threshold will increase the number of patients receiving insulin. We found no significant correlation between the initial glycemia and image quality. We disagree that "the set point to prescribe insulin in the study protocol of Roy et al. might account for their poor image quality." Turcotte et al. used a lower threshold (7.0 mmol/L) and showed no significant increase in muscular uptake (5).

The guidelines of the Society of Nuclear Medicine mention that administering insulin can be considered, although the administration of ${ }^{18}$ F-FDG would have to be delayed after insulin administration (3). This is in keeping with our finding that the interval between insulin and ${ }^{18} \mathrm{~F}-\mathrm{FDG}$ injection is a critical factor for image quality when insulin is administered. ${ }^{18} \mathrm{~F}-\mathrm{FDG}$ biodistribution was adequate in $75 \%$ of patients injected with insulin. This was not a "barely" adequate biodistribution as suggested by the author, but a normal or near-normal biodistribution. Administration of insulin in these patients allowed them to have a diagnostic ${ }^{18}$ F-FDG PET study. Rescheduling PET scans is inconvenient for patients and delays investigation and treatment. Improvement of ${ }^{18} \mathrm{~F}$-FDG biodistribution in the remaining $25 \%$ of patients will certainly require systematic postponing of ${ }^{18} \mathrm{~F}-\mathrm{FDG}$ administration until at least $90 \mathrm{~min}$ after insulin injection.

We entirely agree with Dr. Chiu et al. that a better way to ensure adequate glycemia the day of the PET scan is to "do a "practice run' by checking the patient's blood glucose levels for at least $3 \mathrm{~d}$ before the ${ }^{18}$ F-FDG PET appointment." As we noted in the "Discussion" section of our article, "even with adequate recommendations, some patients will reach the department with elevated glycemia" (1). Calling our protocol a "risky strategy" seems exaggerated. We agree that intravenous insulin requires close medical surveillance, as provided for in our protocol. Six patients experienced hypoglycemia $(9.5 \%)$, as defined by a glycemic level measured at $3.5 \mathrm{mmol} / \mathrm{L}$ or lower by glucometer. Moreover, the 2 patients who presented symptoms responded rapidly to oral glucose. Rescheduling PET is certainly a "no-risk" situation for the PET physician. However, some patients will require a few weeks before being able to reach adequate glycemia. The hypoglycemia risk associated with insulin use should always be balanced with the risk of delayed management. To address the issue of transcellular-shift hypokalemia, we recommended that patients with glycemia above $15 \mathrm{mmol} / \mathrm{L}$ should be rescheduled. We fully agree that there is nonuniform insulin sensitivity among hyperglycemic patients. The aim of this insulin administration, using a sliding scale, was to rapidly control the level of insulin before ${ }^{18} \mathrm{~F}-\mathrm{FDG}$ administration in hyperglycemic patients. It was never intended to manage diabetes or to replace any treatment regimen. In clinical PET practice, the insulin dose should be modulated according to patient profile.
In conclusion, we insist that our study sought to evaluate the safety and effectiveness of insulin administration to reduce glycemia in diabetic cancer patients who display elevated glycemia despite recommendations. We used a pragmatic approach to minimize the need to reschedule patients, reduce the risk of false-negative PET results due to hyperglycemia, and limit the hazards associated with insulin administration in patients with moderately elevated glycemia $(10.0-15.0 \mathrm{mmol} / \mathrm{L})$.

\section{REFERENCES}

1. Roy F-N, Beaulieu S, Boucher L, Bourdeau I, Cohade C. Impact of intravenous insulin on ${ }^{18}$ F-FDG PET in diabetic cancer patients. J Nucl Med. 2009;50:178183.

2. Medina RA, Owen GI. Glucose transporters: expression, regulation and cancer. Biol Res. 2002;35:9-26.

3. Delbeke D, Coleman RE, Guiberteau MJ, et al. Procedure guideline for tumor imaging with ${ }^{18}$ F-FDG PET/CT 1.0. J Nucl Med. 2006;47:885-895.

4. Bombardieri E, Aktolun C, Baum RP, et al. FDG-PET: procedure guidelines for tumour imaging. Eur J Nucl Med Mol Imaging. 2003;30:BP115-BP124.

5. Turcotte E, Leblanc M, Carpentier A, Benard F. Optimization of whole-body positron emission tomography imaging by using delayed 2-deoxy-2-[F-18]fluoroD-glucose injection following i.v. insulin in diabetic patients. Mol Imaging Biol. 2006;8:348-354.

\section{Félix-Nicolas Roy \\ Sylvain Beaulieu \\ Luc Boucher \\ Isabelle Bourdeau \\ Christian Cohade* \\ *Centre Hospitalier de l'Université de Montréal \\ 3840 Saint-Urbain}

Montréal (QC), Canada, H2W 1T8

E-mail: ccohade2000@yahoo.com

DOI: $10.2967 /$ jnumed.109.070540

\section{Influence of Trigger PSA and PSA Kinetics on ${ }^{11} \mathrm{C}$-Choline PET/CT Detection Rate in Patients with Biochemical Relapse After Radical Prostatectomy}

TO THE EDITOR: The Italian investigators recently reported an interesting retrospective study on the effect of total prostatespecific antigen (PSA), PSA velocity, and PSA doubling time on the lesion detection rate of ${ }^{11} \mathrm{C}$-choline PET/CT in 190 men who had been treated with radical prostatectomy and then presented with biochemical failure (1). Similar to a prior study (2), the general conclusion was that ${ }^{11} \mathrm{C}$-choline detection rate increases as the values of the PSA parameters are increased, reflecting the underlying higher disease burden. In particular, the authors reported that the likelihood of lesion detection increases with a trigger PSA higher than $2.4 \mathrm{ng} / \mathrm{mL}$ or in those patients with PSA less than $2.4 \mathrm{ng} / \mathrm{mL}$ when PSA doubling time is lower than $3.4 \mathrm{mo}$ or PSA velocity is higher than $1 \mathrm{ng} / \mathrm{mL} / \mathrm{y}$. However, additional information is needed to decipher the full potential clinical impact of the reported findings. First, the authors do not explicitly provide a definition for biochemical failure. It is assumed that a detectable serum PSA level of at least $0.2 \mathrm{ng} / \mathrm{mL}$ was considered as evidence for biochemical recurrence (PSA relapse), similar to that reported by Pound et al. (3), since this 
value is shown as the minimum PSA in the reported range of PSA levels. It also appears that there was a mixture of patients with PSA relapse only and those with biochemical failure who had other imaging studies with abnormal findings (e.g., bone scan or CT). Despite the notion that ${ }^{11} \mathrm{C}$-choline had a better detection rate than standard imaging, the important clinical question is what the detection rate of nonstandard ${ }^{11} \mathrm{C}$-choline $\mathrm{PET} / \mathrm{CT}$ is in the substantial number of men who present with PSA relapse only when standard imaging studies are negative (by definition). This question is important because currently, the most appropriate diagnostic and therapeutic maneuvers for asymptomatic men with biochemical failure remain undefined (4-6). It is suggested that the results of the report by Castellucci et al. would have been considerably more clinically useful if they had limited their data analysis (or had included the relevant subset of data analysis) to the PSA-relapse-only patients, who at this point cannot be deciphered from the published article. If ${ }^{11} \mathrm{C}$-choline can provide unique information in this specific clinical setting, in which there is currently a void of a viable diagnostic imaging method, then important therapeutic decisions (e.g., salvage local vs. systemic therapy, or both) can be made earlier than when disease becomes apparent on standard imaging, potentially leading to improved patient outcome. Of course, validation of PET findings becomes challenging because by definition there are no standard imaging correlates (7). In such cases, tissue sampling, long-term follow-up, and content validity (e.g., pattern of detected lesions) may serve for validation. The second issue that needs attention is the definition of true-positive PET findings in this study, which was based on visual observation of any focal ${ }^{11} \mathrm{C}$-choline uptake higher than surrounding background levels, correlation to other imaging studies (which we just argued would not be possible if we deal with a restricted definition of biochemical failure with no standard imaging evidence of disease), and regression with therapy or progression with no or ineffective therapy in subsequent scans. However, these validation criteria, as admitted by the authors, are the main limitation of their study. Perhaps these criteria are the reason for no false-positive results with ${ }^{11} \mathrm{C}$-choline PET/CT in this study. For example, decline or resolution of focal uptake does not necessarily mean that a "malignant" lesion responded to treatment, because that lesion may have actually been benign and might have resolved (or improved) regardless of treatment for cancer. Such lesions are in fact false-positives but are labeled truepositives incorrectly simply because of the flawed validation criteria. Finally, it would have been helpful to know if there was a relationship between the PSA parameters and the chance of detecting only local recurrence, only metastatic disease, or both. Clearly additional studies with well-defined groups of patients, validation criteria, and endpoints would be needed in this important clinical setting.

\section{REFERENCES}

1. Castellucci P, Fuccio C, Nanni C, et al. Influence of trigger PSA and PSA kinetics on ${ }^{11} \mathrm{C}$-choline PET/CT detection rate in patients with biochemical relapse after radical prostatectomy. $J$ Nucl Med. 2009;50:1394-1400.

2. Krause BJ, Souvatzoglou M, Tincel M, et al. The detection rate of 11-C choline PET/CT depends on the serum PSA-value in patients with biochemical recurrence of prostate cancer. Eur J Nucl Med Mol Imaging. 2008;35:18-23.

3. Pound CR, Partin AW, Eisenberger MA, et al. Natural history of progression after PSA elevation following radical prostatectomy. JAMA. 1999;281:1591-1597.
4. Moul JW. Prostate specific antigen only progression of prostate cancer. J Urol. 2000;163:1632-1642.

5. Mohile SG, Petrylak DP. Management of asymptomatic rise in prostatic-specific antigen in patients with prostate cancer. Curr Oncol Rep. 2006;8:213-220.

6. Scher HI, Eisenberger M, D'Amico AV, et al. Eligibility and outcomes reporting guidelines for clinical trials for patients in the state of a rising prostate-specific antigen: recommendation from the Prostate-Specific Antigen Working Group. $J$ Clin Oncol. 2004;22:537-556.

7. Apolo AB, Pandit-Taskar N, Morris MJ. Novel tracers and their development for the imaging of metastatic prostate cancer. J Nucl Med. 2008;49:2031-2041.

\section{Hossein Jadvar \\ Keck School of Medicine \\ University of Southern California \\ 2250 Alcazar St., CSC 102 \\ Los Angeles, CA 90033 \\ E-mail: jadvar@usc.edu}

DOI: 10.2967/jnumed.109.071894

REPLY: It is our pleasure to answer the letter of Dr. Jadvar about our paper (1).

The diagnostic flow chart of patients with biochemical relapse after radical prostatectomy has yet to be defined with regard to either the most appropriate test to perform after a prostate-specific antigen (PSA) increase or the optimal timing for performing the test. It is probable that this lack is due to the fact that conventional imaging methods (CT, MRI, bone scanning, transrectal ultrasonography) have shown limited value in restaging of the disease, particularly when the PSA values are low (2). Furthermore, the optimal timing for performing imaging tests after biochemical failure is not well established yet because a balance has to be struck between the clinical need for early detection of relapse and the need to perform the tests when PSA values are high and, consequently, there is a higher probability of detecting relapse (3).

To find a possible solution to this problem, we have tried to clarify at least one aspect: the relationship between PSA values and PSA kinetics on the one hand and ${ }^{11} \mathrm{C}$-choline PET/CT detection rate on the other hand. In response to the principal aim of our study, we can affirm that not only trigger PSA but also PSA kinetics influence PET/CT detection rates.

A secondary aim of our study was to compare the results of PET/CT and other imaging methods such as bone scanning or CT. In our study, of 130 patients who underwent bone scanning before PET/CT, 9 had positive bone scan results and 31 had positive PET/CT results. Furthermore, of 87 patients who underwent CT or MRI before PET/CT, 15 were positive for single lesions, whereas PET/CT detected disease relapse in 29 patients. We did not report PET/CT results for patients in whom the results of all conventional imaging methods were negative: nevertheless, in this context, $12(21.4 \%)$ of 56 patients who showed negative results on conventional imaging showed positive findings on $\mathrm{PET} / \mathrm{CT}$.

The main limitation of our retrospective study is the validation of positive findings, because longitudinal follow-up with PET/CT or conventional imaging is affected by all the limitations identified by Dr. Jadvar. We tried to overcome this critical point by increasing the number of patients enrolled and thus trying to minimize the potential error. To our knowledge, our population is the largest ever studied with PET/CT after biochemical failure (190 patients). In our paper, we reported results on only a patient basis; however, in our population we detected 197 lesions in 74 of 190 
positive patients. We cannot exclude with absolute certainty that a few lesions were erroneously categorized as true-positive; however, considering the high number of lesions analyzed and the high number of patients with multiple lesions, we can assume that the potential presence of a few false-positive lesions would not significantly affect the overall trend of the results.

Finally, to our knowledge this was the first published study taking into consideration the influence of PSA kinetics on PET/CT detection rate. As suggested by Dr. Jadvar, it would also have been useful to investigate the relationship between PSA kinetics and the site of the metastatic lesion. We accept with pleasure this suggestion, which would be the fruitful aim of an additional study.

In conclusion, with our work we hope we have been able to clarify at least some aspects of the possible flow chart to give additional information to clinicians using ${ }^{11} \mathrm{C}$-choline PET/CT in patients with biochemical relapse. The principal objective here is to anticipate the detection of relapse and thus put clinicians in a position to take advantage of a more appropriate and broader set of therapeutic options.

\section{REFERENCES}

1. Castellucci P, Fuccio C, Nanni C, et al. Influence of trigger PSA and PSA kinetics on ${ }^{11} \mathrm{C}$-choline PET/CT detection rate in patients with biochemical relapse after radical prostatectomy. J Nucl Med. 2009;50:1394-1400.

2. Choueiri TK, Dreicer R, Paciorek A, et al. A model that predicts the probability of positive imaging in prostate cancer cases with biochemical failure after initial definitive local therapy. J Urol. 2008;179:906-910.

3. Krause BJ, Souvatzoglou M, Tincel M, et al. The detection rate of 11-C choline $\mathrm{PET} / \mathrm{TC}$ depends on the serum PSA-value in patients with biochemical recurrence of prostate cancer. Eur J Nucl Med Mol Imaging. 2008;35:18-23.

\author{
Paolo Castellucci* \\ Chiara Fuccio \\ Stefano Fanti \\ *University of Bologna \\ Via Massarenti, 9 \\ Bologna 40138, Italy \\ E-mail: paolo.castellucci@aosp.bo.it
}

DOI: 10.2967/jnumed.109.072322 